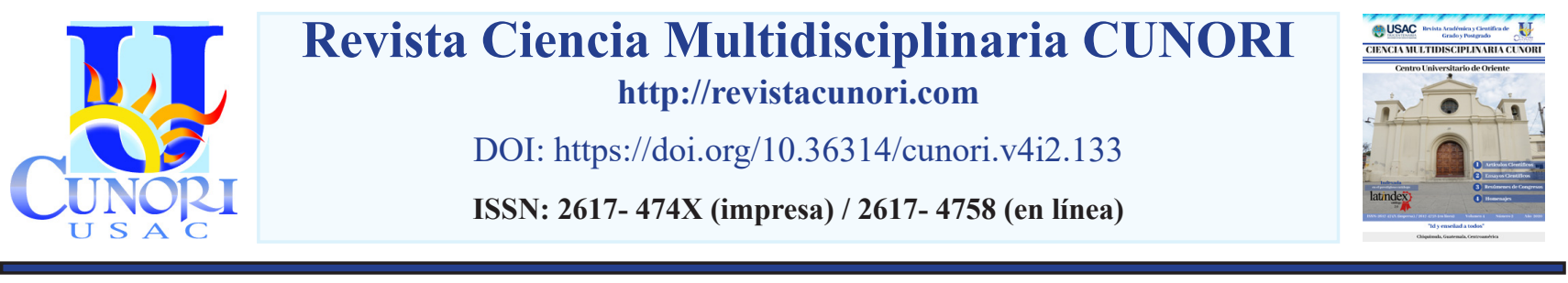

Referencia del artículo

Jarquín-Castro, J. A. (2020). Competencias lectoras de los estudiantes de la carrera de PEM y TAE. Revista Ciencia Multidisciplinaria Cunori, 4(2), 101-107. https://doi.org/10.36314/cunori.v4i2.133

\title{
Competencias lectoras de los estudiantes de la carrera de PEM y TAE
}

\section{Reading competences of the students of the career of PEM y TAE}

\author{
Julio Alfredo Jarquín Castro \\ Universidad de San Carlos de Guatemala \\ Recibido: 13 de septiembre de 2020 / Revisado: 14 de septiembre de 2020 / Aceptado: 14 de octubre de 2020 \\ Disponible en internet el 30 de octubre de 2020 \\ *Autor para correspondencia. \\ Correo electrónico: juliojarcas@gmail.com
}

Resumen

\begin{abstract}
Q1 propósito del manuscrito es dar a conocer los resultados de la tesis Competencias Lectoras de los Estudiantes de la carrera
- de Profesorado de Enseñanza Media y Técnico en Administración Educativa del Centro Universitario de Oriente (CUNORI), en el cual se analizó el nivel de la competencia lectora de los estudiantes de la carrera antes mencionada. La investigación es de tipo descriptiva, con un método hipotético deductivo. Se buscó aceptar o rechazar la hipótesis "Hi Las competencias lectoras que desarrollan los estudiantes de la carrera de Profesorado de Enseñanza Media y Técnico en Administración Educativa del CUNORI, se encuentran en un nivel Medio“, siendo esta la hipótesis que sustenta la investigación. La investigacion se realizó con la población constituida por 295 estudiantes. En la etapa de ejecución se trabajó con una planificación de técnicas e instrumentos, permitiendo desarrollar un enfoque metodológico mixto, concluyendo que se obtuvo resultados puntuales, después de aplicar un test de habilidades y un cuestionario tipo Likert de competencias lectoras, donde se administraron lecturas de comprensión literal, inferencial y crítica. Después de ser sometidos a un proceso de investigación durante dos meses, los estudiantes se ubicaron en el nivel establecido, obteniendo resultados que permitieron confrontar ambas pruebas, aceptando la hipótesis principal, determinando que el nivel de la competencia lectora de los estudiantes de la carrera de Pedagogía nivel técnico del CUNORI esta en un nivel "Medio“, estableciéndose en un 80.0 por ciento, los cuales poseen cierta dificultad en dicho proceso.
\end{abstract}

Palabras clave: competencia lectora, lectura literal, lectura inferencial y lectura crítica

Abstract

$\mathrm{T}$ The purpose of the manuscript is to present the results of the thesis Reading Competences of Students of the career of Teaching Staff of Secondary Education and Technician in Educational Administration of the Eastern University Center (CUNORI), in which the level of competence was analyzed reader of the students of the aforementioned career. The research is descriptive, with a hypothetical deductive method. We sought to accept or reject the hypothesis Hi. The reading competences developed by the students of the CUNORI Teaching Staff of Secondary Education and Technician in Educational Administration are at a Medium level", this being the hypothesis that supports the research. The research was carried out with the universe population, it is constituted by 295 students. In the execution stage, we worked with a planning of techniques and instruments, allowing the development of a mixed methodological approach, concluding that specific results were obtained, after applying a skills test and a Likert-type questionnaire of reading competencies, where readings of literal, inferential and critical understanding. After being subjected to a research process for two months, the students were located at the established level, obtaining results that allowed them to confront both tests, accepting the main hypothesis, determining that the level of reading competence of the students of the career of CUNORI's technical pedagogy is at a "Medium" level, settling at 80.0 percent, which have some difficulty in this process. 
Keywords: reading competence, literal reading, inferential reading and critical reading

\section{Introducción}

En las últimas décadas la lectura constituye según Solarzano, J \& Montero, E. (2011), el punto de partida como el proceso de aprehensión de información y, consecuentemente como interpretación y adopción de una posición determinada respecto a la línea de pensamiento del autor sobre un texto, el cual constituye una necesidad imperiosa para el hombre y el mundo de la cultura, tanto como factor de desarrollo personal, como de desarrollo social. A la posteridad el éxito personal y profesional, se reflejará en las instituciones educativas de todos los niveles, así como de su habilidad para obtener de ellas los mayores beneficios.

La competencia lectora es una de las competencias básicas en el aprendizaje educativo, y se relaciona con el éxito escolar y económico futuro Melgarejo, (2006). En el año 2020 se analizó el nivel de comptencia lectora que poseen los estudiantes cursantes de todos los semestres de la carrera de Pedagogía nivel técnico del CUNORI, en las dimensiones de lectura literal, inferencial y crítica. En tal sentido, la lectura en ámbito de la formación académica es sumamente importante y en el ámbito educativo, especialmente en el nivel superior, es esencial la adquisición de habilidades fundamentales de pensamiento que permita identificar las ideas principales, decodificar adecuadamente los elementos de diversas características de la gama textual, así como a juicios deliberados de las lecturas que realiza.

Las competencias lectoras forman individuos con capacidades de comprender, emplear, reflexionar e interesarse en los textos escritos en un marco contextual. Con el propósito de llevar el proceso de investigación, se plantea la siguiente interrogante ¿Cuáles son las competencias lectoras de los estudiantes de la carrera de Profesorado en Enseñanza Media y Técnico en Administración Educativa del Centro Universitario de Oriente -CUNORI-, de Chiquimula?

La investigación realizada tuvo como objetivo analizar el nivel de competencia lectora de los estudiantes, en su proceso de enseñanza aprendizaje debido que, la formación que se requiere como profesores de enseñanza media es indispensable para el éxito en la sociedad actual y en el contexto donde laboran, y así, poder mejorarlas en función de su práctica docente, favoreciendo el éxito y el fortalecimiento de la lectura en estudiantes de los diferentes niveles educativos del país.

En el estudio se utilizaron diversas técnicas y metodologías para la recopilación y análisis de datos, como la observación, entrevista, la encuesta, análisis de contenidos y los cuestionarios, al observar la influencia que tienen las distintas técnicas e instrumentos, puesto que si son interesantes, las mismas permitirán captar la atención de los estudiantes; para que emitan su opinión en materia textual, tomando en cuenta que en muchas ocasiones el leer provoca en los discentes aburrimiento y lo hacen sin sentido volviendo un proceso tedioso, es decir, que es necesario despertar el interés por la lectura en las aulas universitarias, debido a que el objetivo es identificar el nivel de dominio de la competencia lectora en las tres dimensiones que propone el MINEDUC y PISA-D, y analizar si los sujetos de investigación 
alcanzan dicha competencia e indicar sus debilidades durante el periodo de lectura que se lleva en la aplicación de los instrumentos.

El presente artículo tiene como finalidad puntualizar los resultados obtenidos de la investigación, de las técnicas y metodologías lectoras que poseen los estudiantes en su proceso de formación superior, las cuales tienen cierta dificultad en el desarrollo de lecturas literales, inferenciales y críticas, y a la vez que comprendan que leer es un proceso fascinante, es decir que la lectura permite adentrarse en mundos nuevos de conocimientos, fantasía, aventura y de imaginación y de la grandeza de la vida para descubrir quien es y quien quiere ser.

\section{Materiales y métodos}

Los resultados obtenidos de los instrumentos que determinaron el nivel de desarrollo de las competencias lectoras, definiéndose como la manera en que los estudiantes son capaces de fortalecer la capacidad de autoaprendizaje. En la investigación se tomo en cuenta la totalidad de la población de la carrera, la cual estuvo conforma por 295 estudiantes. El método utilizado fue le hipotético deductivo y las técnicas para la obtención de resultados fueron: la observación, entrevista, la encuesta, análisis de contenidos y los cuestionarios entre otros.

Se realizo un análisis de dominio de los niveles de desarrollo de la competencia lectora en los estudiantes de la carrera de Pedagogía nivel técnico del CUNORI. Como fundamentación teórica se tomo en cuenta el modelo propuesto por el MINEDUC, (2012) y basándose en los criterios PISA-D, (2009), en donde se identifican las dimensiones de lectura literal, inferencial y crítica. Para estudiar y entender el nivel que poseen los estudiantes en sus distintas dimensiones, se utilizo un enfoque mixto (Hernández, S. Fernandez \& Baptista., 2014), el cual representa la recolección y análisis de datos cuantitativos y cualitativos, es decir, que se realizó de forma simultanea, por un lado, el análisis cuantitativo se trabajo a través de un instrumento con escala de Likert y el cualitativo por medio de un test de habilidades de lectura, teniendo como sub variables de estudio la lectura literal, inferencial y crítica.

En este enfoque, Suárez, R. (2001), el planteamiento de hipótesis surge antes de la recolección y análisis de los datos; y cuando se efectúa la recolección de los mismos, se calculan las variables por medio de criterios propuestos por PISA-D, para complementar el análisis de resultados se utilizó para el efecto el programa estadístico SPSS (versión 22.0), los datos obtenidos son el producto de las opiniones de los estudiantes, las cuales sirvieron para comprobar las hipótesis independientes y dependientes planteadas, con base en la medición numérica y el análisis estadístico. Motivo por el cual, la investigación se realizó en grupos ya establecidos, ya que están formados con anterioridad, y los mismos son parte de la investigación, de ellos se obtuvieron los resultados debido a la aplicación de distintas estrategias lectoras, las que fueron evaluadas por medio de test y cuestionarios. El tipo de investigación que se utilizo fue el descriptivo, el mismo busca especificar las propiedades, las características y los perfiles de los discentes que se sometieron al análisis; con un método hipotético deductivo, el cual permitió poner a prueba las hipótesis en el estudio con la finalidad de recoger información y un diseño cuasi experimental, porque difiere de los experimentos "puros”, es decir que los estudiantes no fueron asignados al azar, porque 
los grupos ya existían, ya que surgen de manera independiente o aparte del experimento (Hernández, S. Fernandez \& Baptista., 2014).

Dicho proceso investigativo se realizó durante los meses de junio a julio del año 2020, la población objeto de estudio fueron 295 estudiantes y por las delimitaciones que provoco la pandemia del COVID19, la cuales fueron la suspensión de todas las actividades presenciales académicas dentro de las aulas universitarias, se tuvo que realizar la investigación por medio de cuestionarios digitales elaborados en google forms, los mismos sirvieron para la obtención de los resultados en los semestres restantes.

\section{Estrategias lectoras}

Durante el proceso de investigación se desarrollaron las estrategias lectoras y las mismas fuero: para los niveles de la competencia lectora en las dimensiones de lectura literal, lectura inferencial y lectura crítica; la activación del conocimiento previo, utilización de pistas del contexto, inferencias en el significado, ubicación de palabras claves, organización de texto entre otras.

Tabla No.1 Criterios PISA-D, para determinar el nivel de medición de las competencias lectoras

\begin{tabular}{ll}
\hline Criterios & \multicolumn{1}{c}{ Definición } \\
En el nivel de la comprensión lectora se encuentran los alumnos q \\
pueden leer, en el sentido técnico de la palabra, pero que tien \\
Bajo $\quad$ importantes dificultades para utilizar la lectura como una herramienta q \\
les permita ampliar sus conocimientos y destrezas en diferentes áreas. F \\
lo tanto, está en entredicho su capacidad de beneficiarse de la educaci \\
y aprovechar las oportunidades de aprendizaje durante su vida. \\
En el nivel de la comprensión lectora se encuentran los estudiantes q \\
responden reactivos básicos como los que piden ubicar informaci \\
directa, realizar inferencias sencillas, identificar lo que significa una pal \\
bien definida de un texto y utilizar algunos conocimientos externos pe \\
comprenderla. \\
En el nivel de la comprensión lectora, se encuentran los estudiantes q \\
son capaces de trabajar con reactivos de complejidad moderada. Ubic \\
fragmentos múltiples de información, vinculan distintas partes de un tes \\
y relacionan dicho texto con conocimientos familiares o cotidianos. \\
En el nivel de la comprensión lectora se encuentran los estudiantes q \\
pueden responder a reactivos difíciles, como los que piden ubic \\
información escondida o interpretar significados a partir de sutilezas c \\
lenguaje. Pueden evaluar críticamente un texto.
\end{tabular}

Fuente: Elaboración propia. 
Escala general de los criterios PISA-D, donde se diferencias los niveles de desarrollo de las competencias lectoras, mismo que se utilizo para ubicar el porcentaje de estudiantes por nivel de desarrollo en cada una de las áreas evaluadas.

\section{Resultados}

Tabla No.2 Población estudiantil de la carrera de PEM y TAE del CUNORI

\begin{tabular}{cc}
\hline Semestre & \#Estudiantes \\
\hline I & 92 \\
III & 67 \\
V & 60 \\
VII & 76 \\
Total & 295 \\
\hline
\end{tabular}

Fuente: Elaboración propia, con base en información proporcionada por Control Académico -CUNORI- (2019)

La tabla anterior refleja la población universo de la carrera de Pedagogía nivel técnico, la cual esta conformada por 295 estudiantes y dividida en los semestres cursantes.

Tabla No.3. Nivel de desarrollo de las competencias lectoras en los estudiantes de la carrera de PEM y TAE del CUNORI

\begin{tabular}{|c|c|c|c|c|c|}
\hline \multicolumn{6}{|c|}{ Rango Total Competencias Lectoras } \\
\hline Semestre que cursa & Muy Bajo & Bajo & Medio & Alto & Total \\
\hline \multirow[t]{2}{*}{ Primero } & 7 & 11 & 68 & 6 & 92 \\
\hline & $2.4 \%$ & $3.7 \%$ & $23.0 \%$ & $2.0 \%$ & $31.1 \%$ \\
\hline \multirow[t]{2}{*}{ Tercero } & 0 & 11 & 54 & 2 & 67 \\
\hline & $0.0 \%$ & $3.7 \%$ & $18.0 \%$ & $0.7 \%$ & $22.4 \%$ \\
\hline \multirow[t]{2}{*}{ Quinto } & 0 & 8 & 50 & 2 & 60 \\
\hline & $0.0 \%$ & $2.7 \%$ & $17.0 \%$ & $0.7 \%$ & $20.4 \%$ \\
\hline \multirow[t]{2}{*}{ Séptimo } & 0 & 7 & 64 & 5 & 76 \\
\hline & $0.0 \%$ & $2.4 \%$ & $22.0 \%$ & $1.7 \%$ & $26.1 \%$ \\
\hline \multirow[t]{2}{*}{ Total } & 7 & 37 & 235 & 15 & 295 \\
\hline & $2.4 \%$ & $12.5 \%$ & $80.0 \%$ & $5.1 \%$ & $\begin{array}{c}100.0 \\
\%\end{array}$ \\
\hline
\end{tabular}

Fuente: Elaboración propia con base (SPSS 22.0) 
Con base a los resultados obtenidos en la tabla anterior se apreciar que los estudiantes de la carrera el 80.0 por ciento se sitúan en un nivel "Medio", se evidencia un porcentaje del 12.5 por ciento que se encuentran en un nivel "Bajo", ocupando un mínimo porcentaje se ubican en un 5.1 por ciento en el nivel "Alto" y sin embargo, se aprecia a un pequeño porcentaje (2.4 por ciento) que se ubican en el nivel "Muy bajo" en el desarrollo de la competencia lectora. La hipótesis Hi se acepta, ya que el 80.0 porciento que equivale a un total de 236 estudiantes se encuentran en un nivel de desarrollo "Medio" en la competencia antes mencionada, es decir que, se observa que desarrollan el análisis, interpretación e identificación de la gama textual según los criterios establecidos en la tabla 2.

\section{Discusión}

Para generalizar los datos obtenidos de la aplicación del test de habilidades y cuestionario tipo Likert para obtener el nivel de desarrollo de la competencia lectora, se realizó una suma por discente del total de puntos de cada sub variable para luego tener un punteo total y un nivel general. Y la variable demográfica de "Semestres" la misma se utilizó para determinar los niveles. Según se puede observar, se contabilizaron 295 estudiantes en total. El porcentaje mayoritario de discentes es en el primer semestre con un 31.1 porciento.

Los datos de la tabla 3 dan una percepción de hábitos de lectura que se desarrollan de forma esporádica, creando preocupación por las similitudes encontradas en estudios realizados con anterioridad y en el actual contexto, como el caso de Alvarez, Y. (2012) donde identificó, que los estudiantes de la Universidad de San Carlos de Guatemala no presentan hábitos e interés por la lectura, también Herrarte, E. (2015), ratifica que los discentes de la carrera de PEM y TAE del Centro Universitario de Santa Rosa (CUNSARO), reflejan poca importancia a la comprensión de lectura en su proceso de enseñanza aprendizaje; de igual manera Salas, P. (2012) en su investigación realizada a estudiantes del nivel medio superior de la Universidad Autónoma de Nuevo León México, poseen poca tipología y conocimientos textuales. Concluyendo que, con la carencia de la competencia lectora, el estudiante se limita al nuevo conocimiento misma que seria una competencia importante en el proceso de formación profesional.

\section{Agradecimientos}

Al coordinador, docentes y estudiantes de la carrera de Profesorado de Enseñanza Media y Técnico en Administración Educativa del CUNORI, por el apoyo brindado en la investigación.

\section{Referencias}

Alvarez, Y. (2012), Hábitos de lectura en los estudiantes del noveno semestre de licenciatura en ciencias de la comunicación de la Universidad de San Carlos de Guatemala, (Tesis de Licenciatura). Ciencias de la Comunicación, USAC.

Hernández, S. Fernandez \& Baptista. (2014). Metodología de la Investigación. 6ta. Edición. México D.F.: Mc Graw Hill Education.

Melgarejo, J. (03 de marzo, 2006). La selección y formación del profesorado: clave para comprender el excelente nivel de competencia lectora de los alumnos finlandeses. Revista de Educación, pp. 237-262 
Salas, P. (2012). El desarrollo de la comprensión lectora en los estudiantes del tercer semestre del nivel medio superior de la Universidad Autónoma de Nuevo León (Tesis de Maestría). Universidad Autónoma de Nuevo León, México.

Solarzano, J \& Montero, E. . (2011). "Construcción y validación de una prueba de comprensión de lectura mediante el modelo Rasch". Revista Actualidades Investigativas en Educación., 11(2):1-27. doi: https://doi.org/10.15517/aie.v11i2.10188

Suárez, R. (2001). Metodología de la Investigacion, Diseños y técnicas . Santa Fé, Bogota: McGraw-Hill.

\section{Sobre el autor}

\section{Julio Alfredo Jarquín}

Maestro en Ciencias en Docencia Universitaria con Orientación en Estrategias de Aprendizaje, titulado como Licenciado en Pedagogía y Administración Educativa, egresado del Centro Universitario de Oriente CUNORI. Realiza labor docente en el Instituto Normal para Varones de Oriente INVO de Chiquimula.

Copyright (c) Julio Alfredo Jarquín Castro

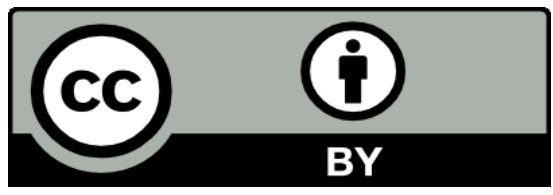

Este texto está protegido por una licencia CreativeCommons 4.0.

Usted es libre para compartir, copiar y redistribuir el material en cualquier medio o formato y adaptar el documento, remezclar, transformar y crear a partir del material para cualquier propósito, incluso comercialmente, siempre que cumpla la condición de atribución: usted debe reconocer el crédito de una obra de manera adecuada, proporcionar un enlace a la licencia, e indicar si se han realizado cambios. Puede hacerlo en cualquier forma razonable, pero no de forma tal que sugiera que tiene el apoyo del licenciante o lo recibe por el uso que hace. 